Skin

Appendage

Disorders
Skin Appendage Disord 2020;6:11-13

DOI: 10.1159/000502700
Received: July 4, 2019

Accepted: August 12, 2019

Published online: September 18, 2019

\title{
Increased Preauricular Wrinkles in Frontal Fibrosing Alopecia Compared to Age-Matched Controls: A Prospective Study of 64 Patients
}

\author{
Sonali Nanda Valeria De Bedout Penelope A. Hirt David E. Castillo \\ Thais Mesquita Laura Scott Mariya Miteva \\ Dr. Phillip Frost Department of Dermatology and Cutaneous Surgery, University of Miami Miller School of Medicine, \\ Miami, FL, USA
}

\section{Keywords}

Frontal fibrosing alopecia · Preauricular lines · Skin aging fibers in biopsies of the preauricular area in diseased patients may contribute. These findings reveal another potential clinical marker of FFA.

(c) 2019 S. Karger AG, Basel

\begin{abstract}
Introduction: Frontal fibrosing alopecia (FFA) is a scarring alopecia affecting mainly postmenopausal females. Associated clinical signs include facial papules, glabellar red dots, depression of frontal veins, and lichen planus pigmentosus. Our objective was to establish the validity of increased preauricular lines as another clinical marker of FFA. Materials and Methods: Thirty-two females with FFA were compared to 32 age-matched females with either androgenetic alopecia or chronic telogen effluvium. Bilateral images of the preauricular area were taken, and disease severity was calculated in all FFA patients using the FFA severity scale (FFASS). The average number of preauricular lines were determined and compared based on group, age, and severity. Results: Patients with FFA had a significantly higher mean number of preauricular lines than controls $(p=0.002)$. Intergroup analysis among the FFA patients revealed no significant difference between FFASS and the number of wrinkles or the number of wrinkles in patients $\geq 60$ years old. Discussion and Conclusion: Females with FFA have increased preauricular lines compared to age-matched controls regardless of age, and disease severity was not correlated to increased lines. Although the cause is unknown, atrophy and loss of elastic
\end{abstract}

() 2019 S. Karger AG, Basel

\section{Introduction}

Frontal fibrosing alopecia (FFA) is a scarring alopecia of complex immunoinflammatory origin. The classic presentation is a band-like alopecia of the frontotemporal hairline extending to the preauricular areas in middleaged females [1]. While caring for FFA patients, we noticed advanced skin aging and sought to determine whether preauricular lines (wrinkles) are increased compared to age-matched controls. Preauricular lines are not related to facial mimics and represent an acceptable marker of intrinsic skin aging.

\section{Methods}

This IRB-approved, prospective, observational study included female patients with FFA and age-matched controls with chronic telogen effluvium (CTE) or androgenetic alopecia seen at the outpatient Dermatology clinic at the University of Miami from October 2018 to February 2019. FFA patients were diagnosed as per the updated criteria [2]. Recorded data included

\section{KARGER}

E-Mail karger@karger.com

www.karger.com/sad
Sonali Nanda, MS

Dr. Phillip Frost of Dermatology and Cutaneous Surgery

University of Miami Miller School of Medicine, 1600 NW 10th Ave

Rosenstiel Science Medical Building, Room 2023A, Miami, FL 33136 (USA)

E-Mail SXN480@miami.edu 

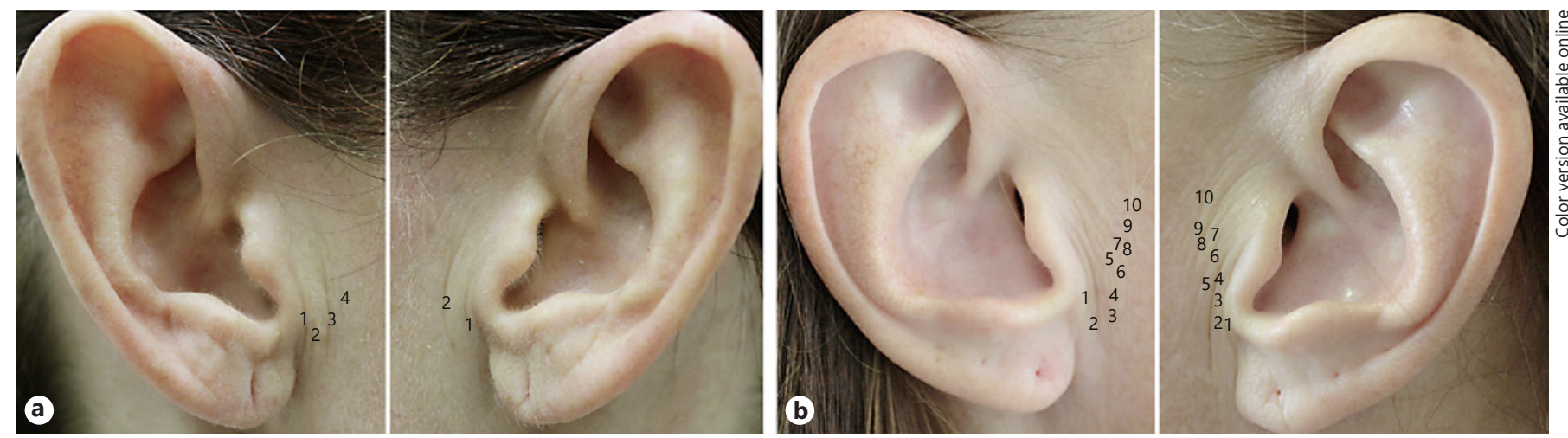

Fig. 1. a The right and left ear of a patient with CTE in her 40s. b The right and left ear of a patient with FFA in her 40s. Note increased preauricular lines on both sides.

Table 1. Patient demographics and results

\begin{tabular}{lccc}
\hline Characteristic & FFA & Control & $p$ value \\
\hline Patients & 32 & 32 & \\
Mean age (SD), years & $59.3(10.8)$ & 60.1 & \\
Race, $n$ (\%) & & & \\
$\quad$ Asian & $4(1.3)$ & $2(3.1)$ & \\
$\quad$ Black & $28(87.5)$ & $29(90.3)$ & \\
$\quad$ Caucasian & & & \\
Ethnicity, $n$ (\%) & $19(59.3)$ & $23(71.9)$ & \\
$\quad$ Hispanic/Latino & $13(40.6)$ & $9(28.1)$ & \\
$\quad$ Not Hispanic/Latino & 7.7 & 4.7 & 0.002 \\
Wrinkles, $n$ & 7.6 & 5.1 & 0.24 \\
$\quad$ Right ear & 7.6 & 4.9 & \\
$\quad$ Left ear & 7 & - & \\
Average number of wrinkles & 8.2 & - & \\
$\quad<60$ years old & 27 & 19 & \\
$\quad>60$ years old & 9.49 & - & \\
Patients $>4$ wrinkles, $n$ & & & \\
Average FFASS $(0-25)$ & & & \\
\hline
\end{tabular}

FFA severity scale (FFASS) scores [3], demographic information, and high-quality images of bilateral preauricular areas and ears. Photographs were taken at the same angle and lighting with a Canon EOS 550D. Patients with a history of smoking or any surgical/cosmetic procedures to the face were excluded. Earrings were removed, and images were cropped by an unblinded investigator (S.N.) who was excluded from the evaluation.

Two blinded dermatologists (M.M., T.M.) counted preauricular lines bilaterally [4] and determined the average per patient. The average number of preauricular lines in FFA patients was compared to control patients and correlated to the FFASS. Data was analyzed using the GraphPad Prism version 6.0. $\chi^{2}$ test, and unpaired $t$ tests were used to compute $p$ values for categorical and quantitative variables, respectively. The significance level was set at 0.05 .

\section{Results}

Thirty-two females with FFA and 32 controls with CTE $(n=18)$ or androgenic alopecia $(n=14)$ completed the study (Table 1). Ages ranged from 33 to 89 years with a mean of 59.7 years (SD 11.2). The distribution by race included Caucasians $(n=57)$, Blacks $(n=6)$, and Asians $(n=1)$, and by ethnicity Hispanic/Latino $(n=42)$, and not Hispanic/Latino $(n=22)$.

Patients with FFA revealed a statistically significant higher mean number of preauricular lines than controls (7.6 vs. $4.9, p=0.002$; Fig. 1 ). FFA was significantly associated with 4 or more wrinkles $(p=0.026)$. Intergroup analysis among the FFA patients revealed no significant 
difference between (1) FFASS and number of wrinkles $(r=0.23, p=0.23)$ and (2) number of wrinkles in patients $<60$ or $>60$ years old $(7.0$ vs. $8.2, p=0.24)$. The average number of wrinkles in the right and left ears of FFA patients were similar (7.68 vs. 7.56$)$.

\section{Discussion}

We observed significantly increased preauricular lines in patients with FFA compared to age-matched controls, including among FFA patients $>60$ years old. Our finding adds to the established clinical signs of FFA that include facial papules, glabellar red dots, depressed frontal veins, and lichen planus pigmentosus [5]. On histopathology, preauricular lines demonstrate actinic elastosis and disappearance of elastic microfibrils and collagen fibers at the dermal-epidermal junction [6]. Although the mechanism for increased preauricular lines is unknown, we speculate that skin atrophy and possibly inflammationinduced degradation of elastic tissue in the upper dermis may contribute. In fact, one study of facial papules in FFA detected reduced and fragmented perifollicular elastic fibers in $92 \%$ of the biopsies [5].

The absence of correlation between FFASS and number of wrinkles, combined with the lack of a significant difference in the number of wrinkles in younger versus older FFA patients supports an intrinsic ageing process that may not be related to actinic sun damage. Further, the left ear, which sustains more sun exposure due to leftside driving in the US, revealed a similar number of wrinkles compared to the right ear.

These findings reveal another potential clinical marker of FFA and may prompt more studies on the underlying disease process.

\section{Acknowledgement}

There are no acknowledgements to make.

\section{Statement of Ethics}

Subjects have given their written informed consent, and the study protocol was approved by the institute's committee on human research (as stated in the manuscript).

\section{Disclosure Statement}

The authors have no conflicts of interest to declare.

\section{Funding Sources}

The authors did not receive any funding for this research.

\section{References}

1 Rossi A, Grassi S, Fortuna MC, Garelli V, Pranteda G, Caro G, et al. Unusual patterns of presentation of frontal fibrosing alopecia: A clinical and trichoscopic analysis of 98 patients. J Am Acad Dermatol. 2017 Jul;77(1):172-4.

2 Vañó-Galván S, Saceda-Corralo D, MorenoArrones OM, Camacho-Martinez FM. Updated diagnostic criteria for frontal fibrosing alopecia. J Am Acad Dermatol. 2018 Jan; 78(1):e21-2.
3 Saceda-Corralo D, Moreno-Arrones OM, Fonda-Pascual P, Pindado-Ortega C, Buendía-Castaño D, Alegre-Sánchez A, et al. Development and validation of the Frontal Fibrosing Alopecia Severity Score. J Am Acad Dermatol. 2018 Mar;78(3):522-9.

4 Lemperle G, Holmes RE, Cohen SR, Lemperle SM. A classification of facial wrinkles. Plast Reconstr Surg. 2001 Nov; 108(6):173550.
5 Pirmez R, Barreto T, Duque-Estrada B, Quintella DC, Cuzzi T. Facial Papules in Frontal Fibrosing Alopecia: Beyond Vellus Hair Follicle Involvement. Skin Appendage Disord. 2018 Aug;4(3):145-9.

6 Bosset S, Barré P, Chalon A, Kurfurst R, Bonté $\mathrm{F}$, André $\mathrm{P}$, et al. Skin ageing: clinical and histopathologic study of permanent and reducible wrinkles. Eur J Dermatol. 2002 MayJun;12(3):247-52. 